\title{
Extending Behavioral Models to Generate Mission-Based Driving Cycles for Data-Driven Vehicle Development
}

Sogol Kharrazi, Marcus Almen, Erik Frisk and Lars Nielsen

The self-archived postprint version of this journal article is available at Linköping University Institutional Repository (DiVA):

http://urn.kb.se/resolve?urn=urn:nbn:se:liu:diva-15.5012

N.B.: When citing this work, cite the original publication.

Kharrazi, S., Almen, M., Frisk, E., Nielsen, L., (2019), Extending Behavioral Models to Generate Mission-Based Driving Cycles for Data-Driven Vehicle Development, IEEE Transactions on Vehicular Technology, 68(2), 1222-1230. https://doi.org/10.1109/TVT.2018.2887031

Original publication available at:

https://doi.org/10.1109/TVT.2018.2887031

Copyright: Institute of Electrical and Electronics Engineers (IEEE)

http://www.ieee.org/index.html

(C)2019 IEEE. Personal use of this material is permitted. However, permission to reprint/republish this material for advertising or promotional purposes or for creating new collective works for resale or redistribution to servers or lists, or to reuse any copyrighted component of this work in other works must be obtained from the IEEE. 


\title{
Extending behavioral models to generate mission-based driving cycles for data-driven vehicle development
}

\author{
Sogol Kharrazi, Marcus Almén, Erik Frisk, Lars Nielsen
}

\begin{abstract}
Driving cycles are nowadays to an increasing extent used as input to model-based vehicle design and as training data for development of vehicles models and functions with machine learning algorithms. Recorded real driving data may underrepresent or even lack important characteristics, and therefore there is a need to complement driving cycles obtained from real driving data with synthetic data that exhibit various desired characteristics. In this paper, an efficient method for generation of mission-based driving cycles is developed for this purpose. It is based on available effective methods for traffic simulation and available maps to define driving missions. By comparing the traffic simulation results with real driving data, insufficiencies in the existing behavioral model in the utilized traffic simulation tool are identified. Based on these findings, four extensions to the behavioral model are suggested, staying within the same class of computational complexity so that it can still be used in large scale. The evaluation results show significant improvements in the match between the data measured on the road and the outputs of the traffic simulation with the suggested extensions of the behavioral model. The achieved improvements can be observed with both visual inspection and objective measures. For instance, the $40 \%$ difference in the relative positive acceleration (RPA) of the originally simulated driving cycle compared to real driving data was eliminated using the suggested model.
\end{abstract}

Index Terms-Powertrain dimensioning, representative driving cycles, real driving data, driver model, machine learning.

\section{INTRODUCTION}

$\mathrm{D}$ RIVING cycles, which are represented by vehicle speed versus time, are commonly used to characterize the vehicle usage in real traffic. The use of driving cycles has a long history, primarily for legal homologation and engine emission certification. Examples of such driving cycles are NEDC, used for certification of passenger cars in Europe, and FTP-75 which is used in the US [1]. Questions about the representativeness of these driving cycles, have resulted in development of a new test procedure (WLTP) using the Worldwide Harmonized Light Vehicle Test Cycle (WLTC), which is an ensemble of three

Manuscript received xxxx xx, 2018; revised xxxx xx, 2018; accepted xxxx xx, xxxx. Date of publication xxxx xx, xxxx. Copyright (c) 2015 IEEE. Personal use of this material is permitted. However, permission to use this material for any other purposes must be obtained from the IEEE by sending a request to pubs-permissions@ieee.org.

S. Kharrazi, E. Frisk, and L. Nielsen are with the Department of Electrical Engineering, Linköping University, 58183 Linköping, Sweden. S. Kharrazi driving cycles adapted to vehicles with three different power to mass ratio [2]. WLTC, in contrast to NEDC, is developed using real driving data gathered around the world and has been in effect in the European Union since 2017.

Still, single homologation driving cycles such as NEDC and WLTC are not sufficient for representing the vehicle usage in real traffic under various conditions. The advent of more complex vehicle designs has resulted in a need for easy access to a multitude of representative driving cycles, which is crucial for dimensioning and optimizing vehicle components and functions. One approach since several years is to use stochastic methods, like clustering and Markov chains, to generate driving cycles by assembling recorded speed profiles from large databases of real driving data. A few examples of driving cycles developed by stochastic methods are the ARTEMIS European driving cycles, which are derived from driving data using automatic clustering tools [3]. The driving cycle constructed by Lin \& Niemeier [4] based on the data for California's regulatory cycle is another example. An alternative stochastic process for construction of driving cycles from real driving data, utilizing Markov chains and transition probability matrices, is presented in [5]. Statistical approaches have also been used for generation of driving cycles specific for hybrid vehicles [6]. Construction of representative driving cycles with equivalent properties is investigated in the work by Nyberg et al. [7, 8]. Higher order and multidimensional Markov chains have been used to improve the representativeness of the generated driving cycles in $[9,10,11]$.

The above-mentioned methods are very useful but considering that certain characteristics may be lacking or underrepresented in the recorded databases, the resulting driving cycles cannot meet the extra demands set by the recent trends of data-driven development of models and functions for vehicle design. In these circumstances there is a need for generation of driving cycles that exhibit various characteristics, which may be missing or unbalanced in recorded data. Such synthetic driving cycles are needed to complement the training data when using machine learning for development of models and functions, to ensure robust vehicle designs that can handle various driving behaviors and conditions.

also works at the Swedish National Road and Transport research Institute, Linköping, Sweden. (e-mail: sogol.kharrazi@ vti.se). M. Almén was a student with the Department of Electrical Engineering, Linköping University. He is now with Saab Defense \& Space, Linköping, Sweden (email: marcus.almen1@saabgroup.com).

This work was supported by the Swedish Electromobility center. 
The main scope of this paper is to develop an efficient method for generation of such synthetic driving cycles. To do so, the concept of mission-based driving cycles is used, which allows a natural way of inclusion of different driving conditions, and also allows easy comparison with real driving data. By generating customized mission-based driving cycles, the influential factors on driving patterns such as street type, obstacles, interfering traffic, and driver behavior, can be altered as desired to exhibit different required characteristics.

To generate mission-based driving cycles, an appropriate method for simulation of driving missions and suitable driver/vehicle models are needed. Simulation of driving missions is an underexplored research topic, however significant knowledge and experience can be extracted from the field of traffic simulation. In the literature, a few case studies on utilizing traffic simulation for estimating emissions and simulating driving cycles can be found in $[12,13]$.

This paper explores feasibility of generation of missionbased driving cycles by traffic simulation. More specifically, it looks into the existing driver/vehicle models in traffic simulation tools, commonly referred to as behavioral models, and investigates how they can be extended, and analyses its effect on the resulting mission-based driving cycles. Before describing the developed methodology and achieved results, a brief introduction to traffic simulation is provided in the following section.

\section{TRAFFiC SimULATION}

Traffic simulation is a cost-efficient means of investigating vehicles movements in a traffic system. Traffic simulation tools include models for describing the vehicle/driver behavior, as well as interaction between the vehicles and with the infrastructure. Traffic simulation models are typically classified according to the level of detail at which they represent the traffic stream. For instance, macroscopic models use a low level of detail, both with regard to the representation of the traffic stream and interactions between vehicles; while microscopic models represent the traffic stream with higher level of detail and describe individual vehicles and the interaction between them. The possibility of modelling the interaction of the ego vehicle/driver with the surrounding traffic is of key importance for generation of mission-based driving cycles, which makes microscopic traffic simulation an attractive choice for simulating driving missions.

Microscopic traffic simulations have traditionally been used to evaluate different road designs and traffic management strategies. They have also been used to investigate the impact of driver assistance systems, examples of which can be found in [14]. In microscopic traffic simulation, a driver and a vehicle are usually modelled as one unit, using behavioral models. Different behavioral models are incorporated in microscopic simulations to describe how the vehicles perform, how they are driven, and how they interact with each other as well as with the infrastructure. Examples of existing behavioral models are speed adaptation, car following, lane changing, overtaking and gap acceptance; the variation among the drivers in these models is generally described by a distribution function [15]. A review of behavioral models in microscopic traffic simulations can be found in [16].

\section{METHOD}

The first step for investigating the feasibility of generation of mission-based driving cycles by traffic simulation was to compare real driving data, gathered for a specific driving mission, with outputs of simulating the same driving mission. Based on the differences between real driving data and traffic simulation results, potential improvements of the behavioral models were identified. Consequently, the existing behavioral models were extended, which resulted in significant improvements in the match between traffic simulation results and real driving data.

\section{A. Traffic Simulation Tool}

In this study, a free open source microscopic traffic simulation package, called SUMO (Simulation of Urban Mobility), is used [17]. In SUMO, each vehicle is defined explicitly and has a defined route through the network. The default behavioral model in SUMO is a modification of the model defined by Krauß [18] which guarantees the safety of vehicles on the road by including a safety distance between them. In this model, the vehicle speed, $v$, at each time step is decided based on a safe speed, $v_{\text {safe }}$, which prevents collision with the leading car with a speed of $v_{1}$, the maximum acceleration capability of the vehicle, $a$, and a desired speed, $v_{\text {des }}$, which can be interpreted as the driver desired speed based on the speed limit. The model can be formulated as:

$v_{\text {safe }}(t)=v_{1}(t)+\frac{g(t)-g_{\text {des }}(t)}{\tau+\frac{v_{1}(t)+v(t)}{2 b}}$
$v(t+\Delta t)=\min \left[v(t)+a(v) \Delta t, v_{\text {safe }}(t), v_{\text {des }}\right]$

where $g, g_{\text {des }}$ are the actual and desired distances between the ego vehicle and its leader. Symbol $\tau$ represents the reaction time of the driver-vehicle unit, and $b$ is the maximum deceleration of vehicle.

Some of the parameters of the default behavioral model can be assigned by the user to tune the driver characteristic, such as maximum acceleration and deceleration limits and vehicle expected multiplicator for speed limits.

\section{B. Sample Driving Mission}

To compare real driving data and traffic simulation results, a driving mission with available gathered data on road, was simulated in SUMO. The driving mission included both urban and rural sections, as well as a $90 \mathrm{~km} / \mathrm{h}$-road highway, see Fig. 1. Twelve different drivers had driven through this route with an instrumented car. Data from a GPS navigator, an inertial measurement unit, as well as selected CAN messages were logged for each test drive.

The same driving mission was simulated in SUMO, by importing the road network from OpenStreetMap. The SUMO default behavioral model was used for the ego vehicle, as well as the other cars in the traffic. However, for a better comparison between simulation results and the data measured on the road, the parameters of the behavioral model for the ego vehicle were chosen based on the averaged driving characteristics of each test person. The tuned parameters were maximum acceleration and deceleration limits and the vehicle expected multiplicator for speed limits. 


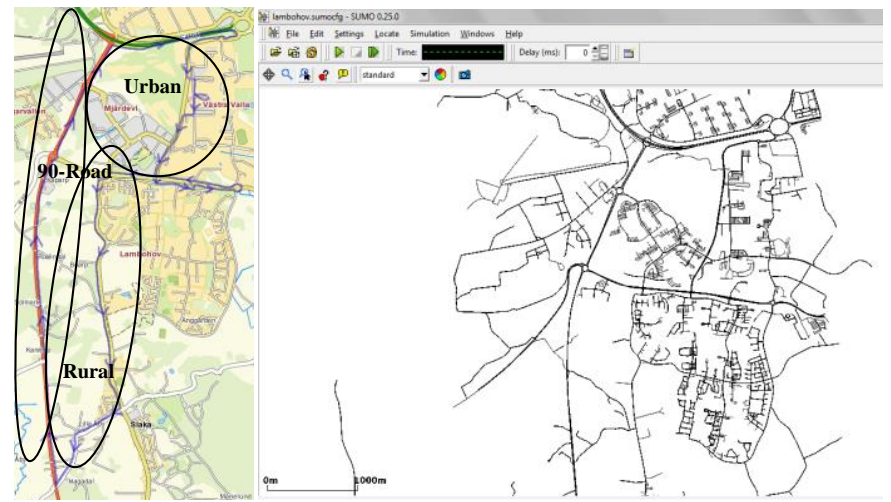

Fig. 1. Driven route (left) and the corresponding simulated road network (right)

\section{DIFFERENCES BETWEEN TRAFFIC SIMULATION RESULTS AND REAL DRIVING DATA}

The results of the traffic simulation with no interfering traffic, versus the corresponding real driving data for three test persons, are plotted in Fig. 2. The general driving patterns in the test data and simulation results are similar and it is possible to distinguish between aggressive and calm drivers in both. This result illustrates the potential of traffic simulation for generation of mission-based driving cycles. However, the existing behavioral models in traffic simulation have been mainly developed for studies on traffic management strategies, and therefore are not sufficiently detailed for capturing all driving behaviors observed in the real driving data. Main driving behaviors which are not captured by the SUMO behavioral model, highlighted in Fig. 3, are:

1. The behavioral model in SUMO does not adapt the speed to the road curvature as human drivers do. For example, left plot in Fig. 3 shows a tight curve at the $7 \mathrm{~km}$ distance with radius of approximately $35 \mathrm{~m}$. The simulated drivers, in contrast to real drivers, do not decrease speed and follow the speed limit of the road.

2. The simulated results show a monotonous speed along the road and lacks the natural speed variation which is visible in the test data.

3. In the simulation the maximum braking is always applied, while in real driving drivers might coast or gradually decrease the speed during braking.

4. The simulated drivers always apply maximum acceleration, while in real driving acceleration changes depending on the speed and situation.

\section{DEVELOPMENT OF BEHAVIORAL MODELS}

Comparison of real driving data with traffic simulation results illustrated that there is a need for improvement of the existing behavioral models to generate mission-based driving cycles that better resembles the real driving data. This section describes the developed extensions to the behavioral model. It should be noted that the goal was to keep the same level of model complexity to avoid increasing the computational complexity of the traffic simulation, so that it can still be used in large scale.

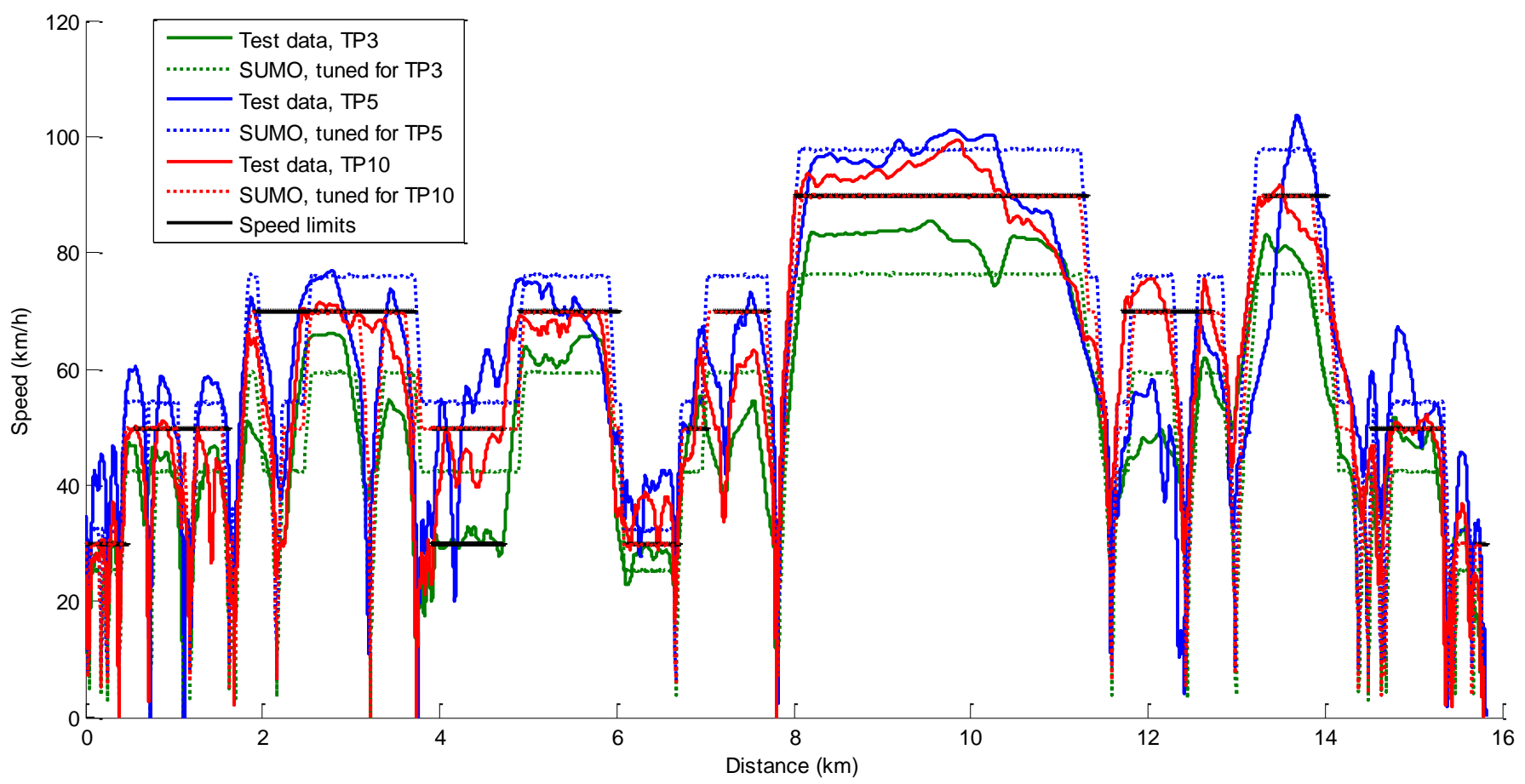

Fig. 2. Speed profiles of three Test Persons (TP) versus corresponding results from microscopic traffic simulation. Note: the road section at the distance of 4-5 km has two speed limit lines; since, in addition to the speed limit sign of $50 \mathrm{~km} / \mathrm{h}$, there was a recommended speed sign of $30 \mathrm{~km} / \mathrm{h}$. 

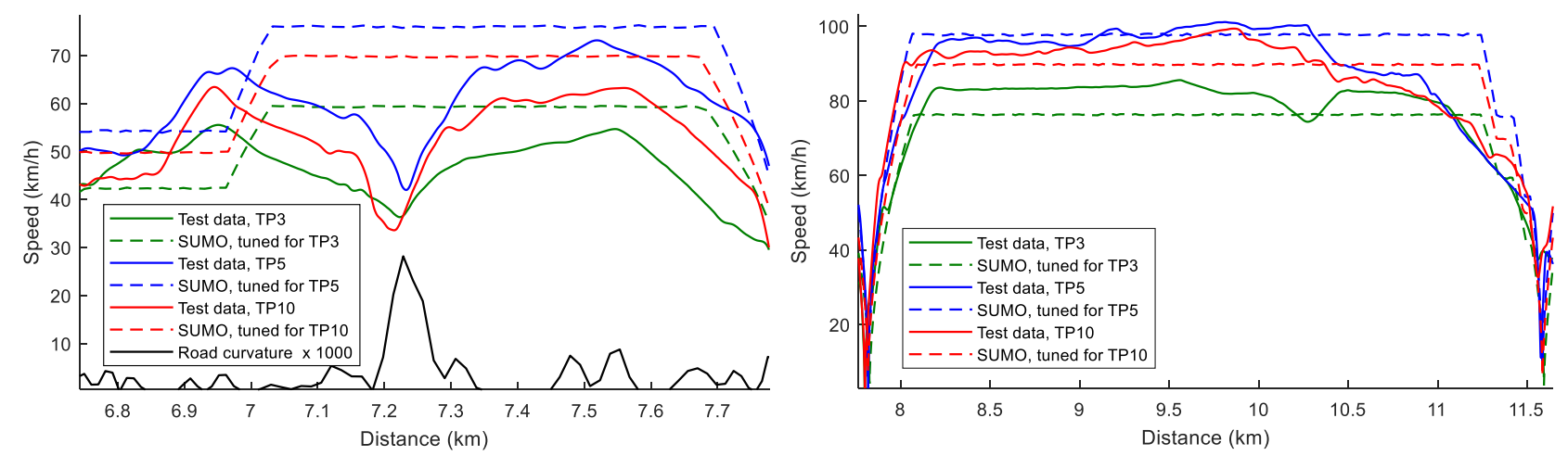

Fig. 3 Speed adaptation of the test persons to the road curvature can be observed in the test data, but it is missing in the simulation results (Left) The simulation results also lack natural speed variation, different acceleration levels and gradual deceleration of drivers, which is visible in the test data (Right).

\section{A. Gathering Additional Driving Data}

To improve the traffic simulation behavioral model with respect to the identified insufficiencies, extra real driving data on a route with a considerable number of curves and intersections, which also includes highway driving, was needed. Therefore, an additional route for data collection, with the same test car as in the initial study, was selected. This route (referred to as route 2) is $19 \mathrm{~km}$ long, includes six traffic lights, around 30 significant curves and approximately $5 \mathrm{~km}$ of highway driving, and was driven by 15 different drivers, see Fig. 4. Route 2 is quite flat so any speed fluctuations due to slopes is negligible.

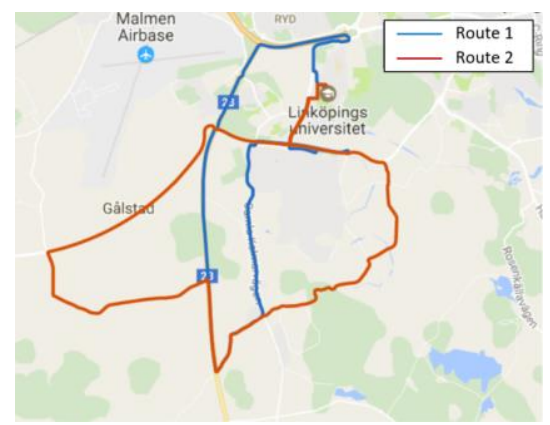

Fig. 4. Chosen route for new measurements (Route2) and the old one (Route1)

\section{B. Learning from Real Driving Data}

Data-driven models, such as linear regression and neural networks, were used to build behavioral models by learning and predicting from the gathered real driving data. Other examples of research on machine learning for driver behavior recognition and prediction can be found in $[19,20]$.

It should be noted that due to the limited real driving data at hand, the achievable improvement in the behavioral model in this study was restricted. However, the objective of this study is to show and compare the effect of improved behavioral model versus the default behavioral model in SUMO on the resulting mission-based driving cycles; thus, the perfection of developed behavioral model is not essential.

To improve the driving behavior realism in the traffic simulation with respect to identified insufficiencies, four extensions to the default behavioral model were developed in Matlab:

1. Speed adaptation to the road curvature

2. Speed variation in free driving

3. Gradual braking

4. Acceleration dependency on speed/gear

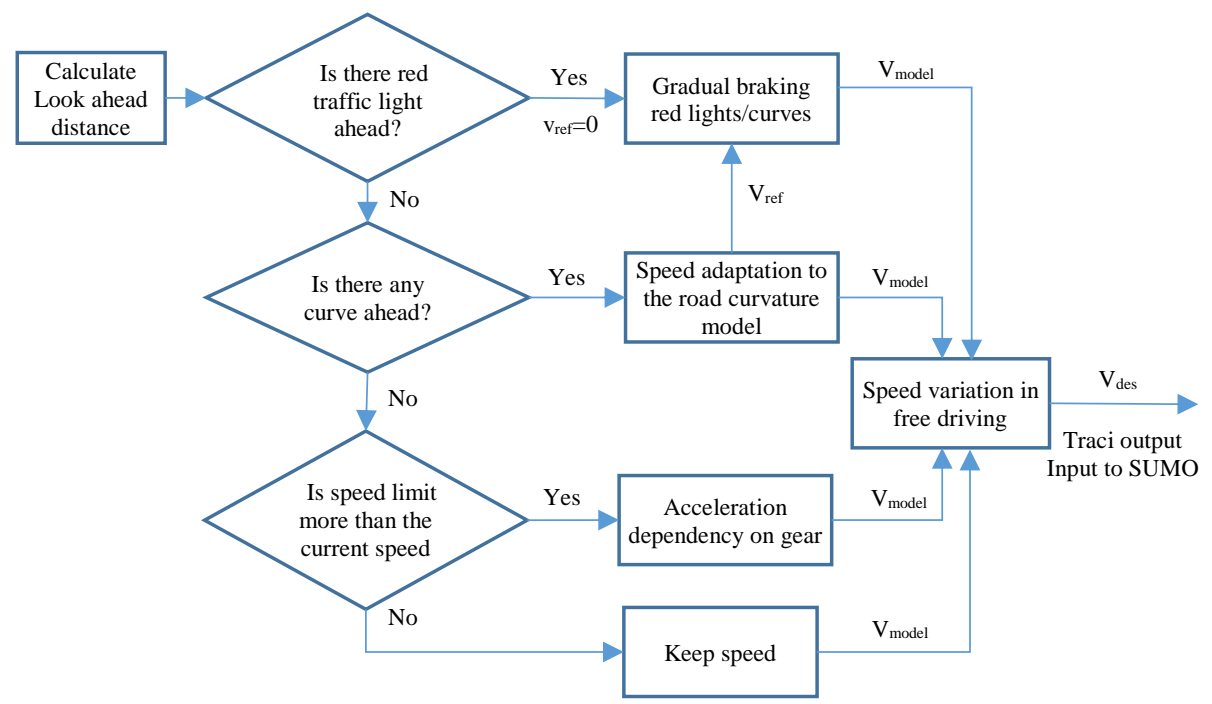

Fig. 5. Layout of the functions of the developed extensions to the default behavioral model 
Additionally, to detect when to activate the developed extensions of the behavioral model, a look-ahead model was developed. The look-ahead model calculates the distance ahead which should be checked for curves or other obstacles, such as red lights.

The vehicle's car-following behavior should still be adhered, when using the new extensions, to avoid collisions. Thus, only $v_{\text {des }}$ in (1) is adjusted with these models. The speed adjustment is communicated with SUMO, via a Matlab toolbox called Traci, Traffic control interface, [21]. Fig. 5 illustrates how the developed extensions of the behavior model are connected to each other and to SUMO. Further descriptions are provided in the following sections.

\section{Look-ahead Distance}

The look-ahead distance, or braking distance, depends on the current speed and the desired change in speed. Note that for a red light the desired change in speed is equal to the current speed, since the vehicle should come to a complete stop. But for a curve, the desired change in speed can vary depending on the curvature. The look-ahead distance increases for higher value of speed and speed change, and a quadratic function can be used to describe it:

$f_{\text {distance }}(v, \Delta v)=a_{1} v+a_{2}\left(v \Delta v-0.5 \Delta v^{2}\right)$

Using linear regression, the model variables, $a_{1}$ and $a_{2}$ were estimated to be 2.72 and 1.49 , respectively. The braking distances in speed profiles of individual drivers were identified and used as input/validation data for the model. To do so, local minima in the speed profiles were found, representing end of braking, see Fig. 6. By analyzing the longitudinal acceleration, a point prior each local minimum in the speed profile was identified as the point where braking initiated. This procedure resulted in approximately 500 samples of braking distances with different starting and end speeds. It should be noted that the modeling was simplified by considering one model for all the drivers, despite different driving patterns. The model fit is shown in Fig. 7.

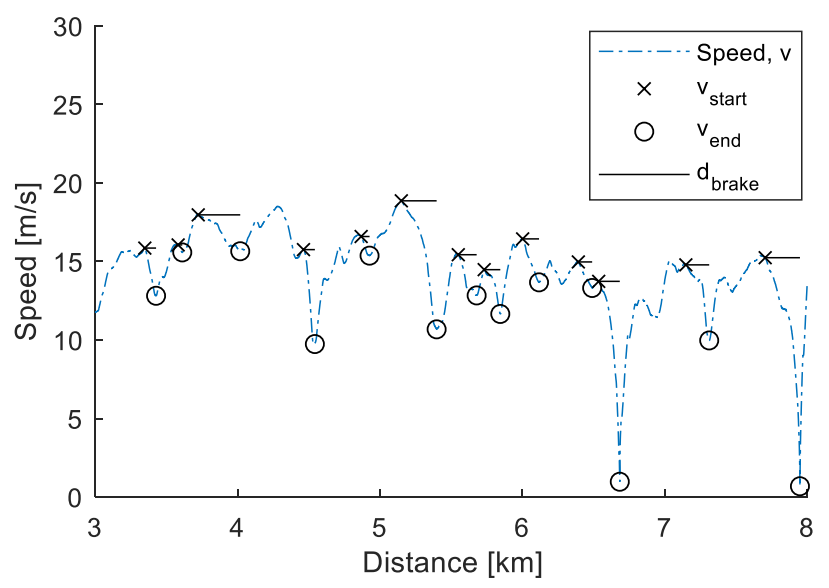

Fig. 6. Sample data used for look-ahead distance model

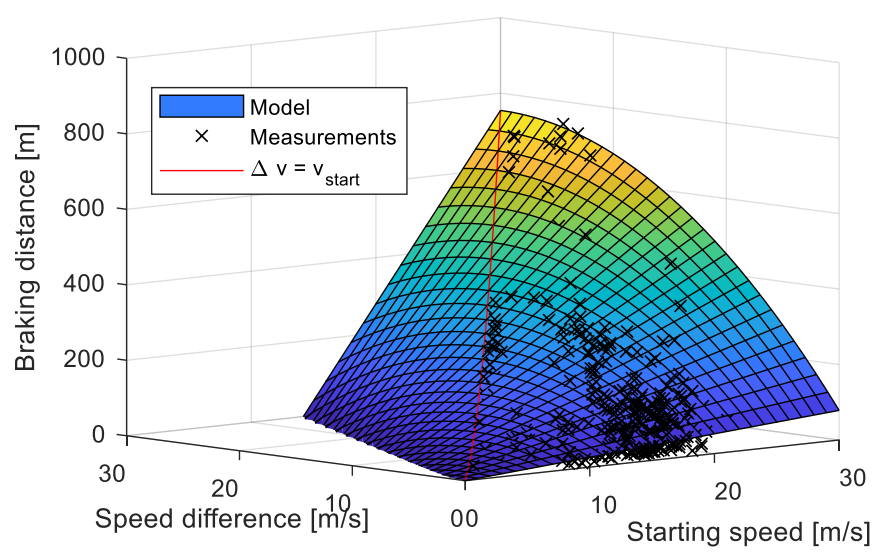

Fig. 7. The look-ahead distance model versus measurement data

\section{Speed Adaptation to the Road Curvature}

To develop a model for the driver speed adaptation to the curve, it was assumed that there is a desired speed through a curve depending on the curvature. This desired speed was calculated from the mean speed profile of all drivers. It should be noted that the speed profiles of some of the test drivers were not considered when calculating the mean, due to disturbances from other vehicles on the road. This was to ensure that only speed adaptation to the infrastructure, namely road curvature, was considered.

To identify the desired speeds for each curve, points of local maxima in the road curvature along the route were matched to local minima in the mean speed profile, see Fig. 8. In total, 30 curves were identified along the Route 2 . The desired speed through a curve does not only depend on the peak curvature, $\kappa$, but also on how the curvature evolves; thus, the curvature integral with respect to distance, $\kappa_{\text {int }}$, within $15 \mathrm{~m}$ on each side of the peak curvature position, $\kappa_{\text {pos }}$, was used for modeling, described as:

$\kappa_{\text {int }}=\int_{\kappa_{\text {pos }}-15}^{\kappa_{\text {pos }}+15} \kappa(x) d x$

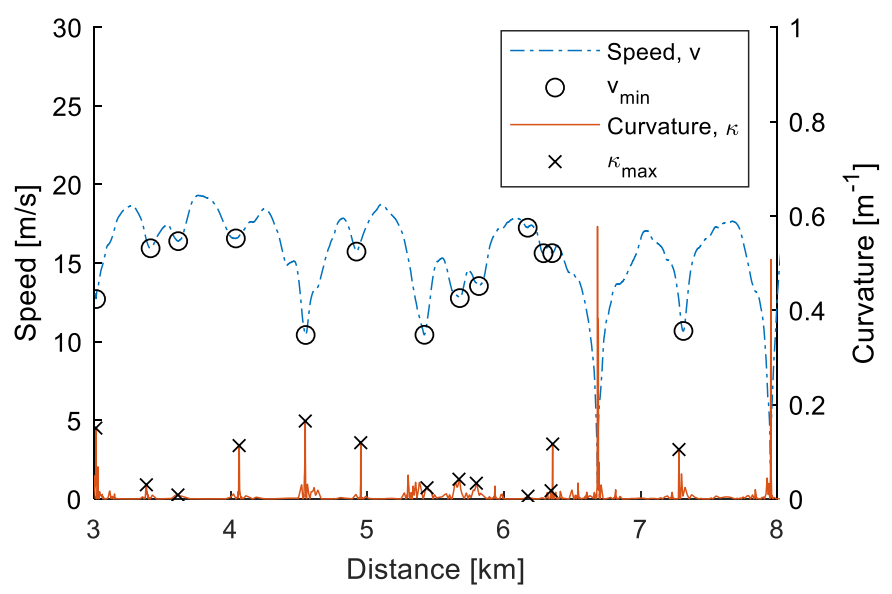

Fig. 8. Sample data used to model desired speed in curves 

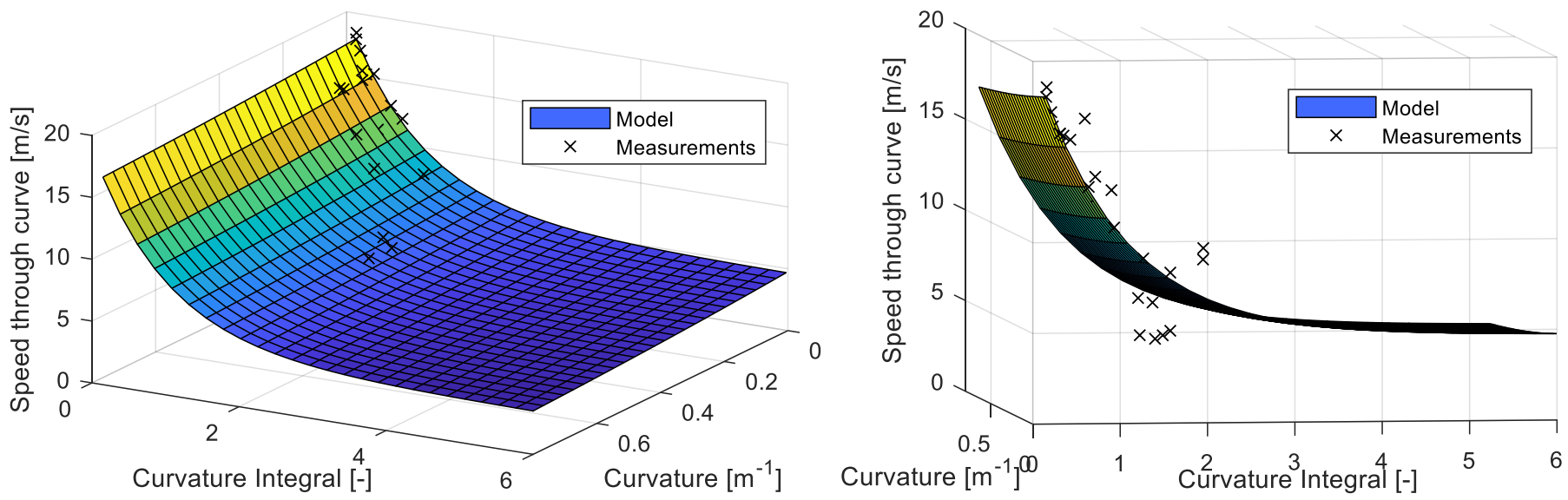

Fig. 9. Model of speed adaptation to the road curvature versus measurements, plotted from two different views.

It should be noted that $\kappa(x)$ is the absolute value of the curvature. An exponential function of the curvature integral and the peak curvature was selected as the candidate model describing the tendency to decrease speed by increasing curvature, formulated as:

$f_{\text {curve }}\left(\kappa, \kappa_{\text {int }}\right)=b_{1}+b_{2} e^{-\kappa}+b_{3} e^{-\kappa_{\text {int }}}$

Linear regression was used to estimate the model variables, $b_{1}, b_{2}$ and $b_{3}$ to $2.27,2.48$ and 15.40 respectively.

The model fit to the data, shown in Fig. 9 is reasonably good and captures the general pattern of the measurements. The resulting model show that the curvature integral, i.e., overall curvature around the peak is a more important factor than the peak curvature value for the desired speed through the curve.

\section{E. Speed Variation in Free driving}

The speed variation during free driving was modelled as a stochastic process, replicating the drivers' behavior on the road; a Wiener process was used for this purpose. A Wiener process is a stochastic process where each sample is a random increment on the previous sample. The increments are taken from an independent sequence of Gaussian normal distribution with zero mean [22]. To ensure that the noise does not deviate too far from zero, a threshold, $b$, was set, above which the noise was sampled from a distribution with a non-zero mean to bring it back within the threshold. The noise was implemented in the model in proportion to the desired speed, $v_{\text {des }}$.

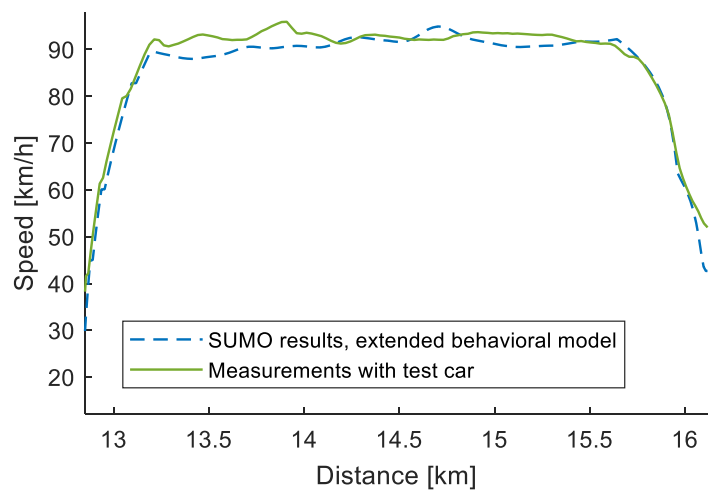

Fig. 10. Simulation results with the extended behavior model
The complete process can be described as follows:

$$
\begin{aligned}
\mu_{k} & =\left\{\begin{array}{cc}
0 & \left|\omega_{k-1}\right|<b \\
a\left(b \operatorname{sign}\left(\omega_{k-1}\right)-\omega_{k-1}\right) & \text { otherwise }
\end{array}\right. \\
x_{k} & =\mathcal{N}\left(\mu_{k}, \sigma^{2}\right) \\
\omega_{k} & =\omega_{k-1}+x_{k} \\
v_{k} & =v_{\text {des }}\left(1+\omega_{k}\right)
\end{aligned}
$$

The standard deviation, $\sigma=0.001$, the threshold, $b=0.05$, and the gain, $a=0.01$, are the model parameters and were tuned to get a good visual match with the real driving data, see Fig. 10.

\section{F. Braking Profile Model}

To model the braking profile, same data intervals identified for modeling the look ahead/braking distance were used. The braking profile is defined as deceleration changes through a brake over a given distance for a certain speed reduction. Looking at the braking profiles, no clear qualities for selection of a well-defined function could be observed, e.g. Fig. 11 shows 5 braking profiles used as input for modeling, out of 300 . Thus, an artificial neural network was used for function fitting. Two separate functions were developed, one for braking before red traffic lights, and another one for braking through curves.

The neural network that was considered for the braking profile models is a small feedforward neural network consisting of an input, a hidden layer with 6 number of neurons, and an output layer that produces the function output [23]. The neuron functions for the hidden layer are sigmoid transfer functions and the output layer contains one neuron with a linear transfer function, see Fig. 12. This type of neural network can approximate any function given enough data and a sufficient number of neurons in the hidden layer [24]. The data was divided into training and validation sets with $80 \%-20 \%$ distribution. The Levenberg-Marquardt algorithm for nonlinear estimation was used to train the model $[25,26]$. 

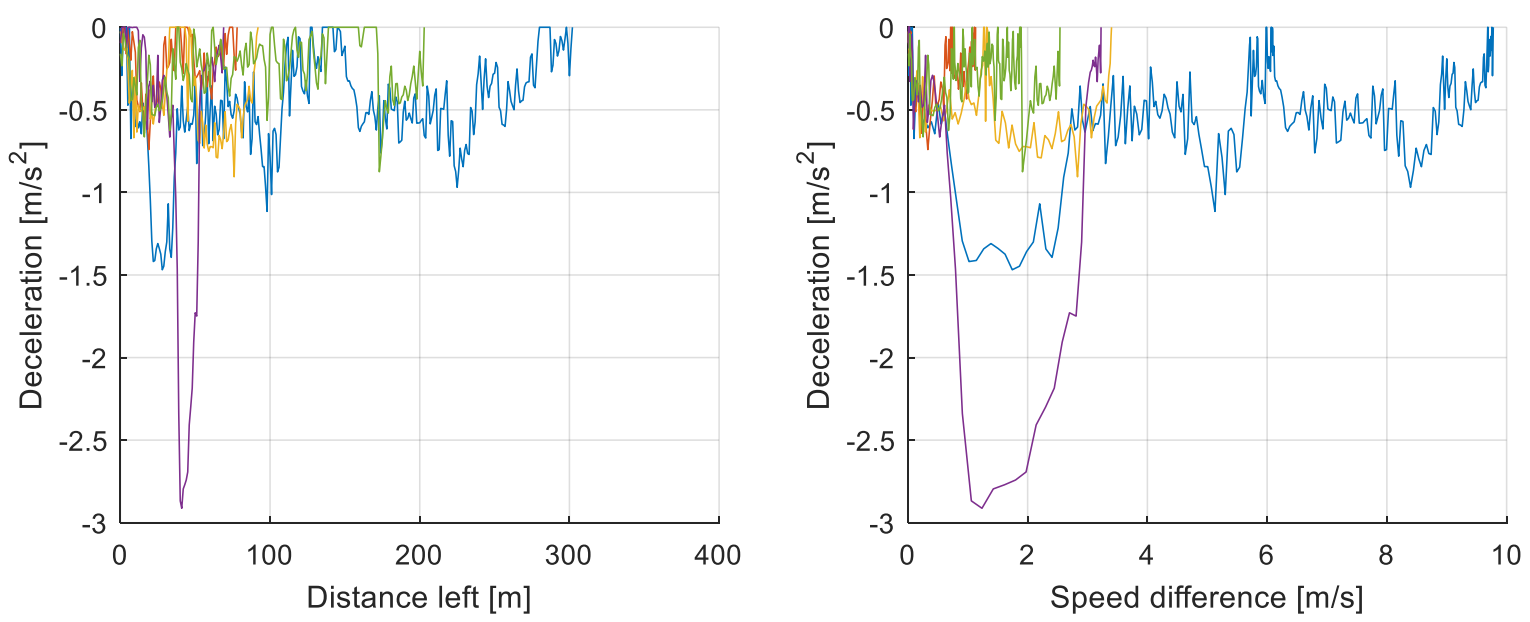

Fig. 11. Sample braking profiles, used as input for function fitting with an artificial neural network

The achieved braking profile model for curves is shown in Fig. 13. As expected the absolute value of deceleration increases with larger desired speed reduction and shorter distance. As the distance to the curve decreases, the effect of desired speed reduction on the deceleration value becomes more prominent.

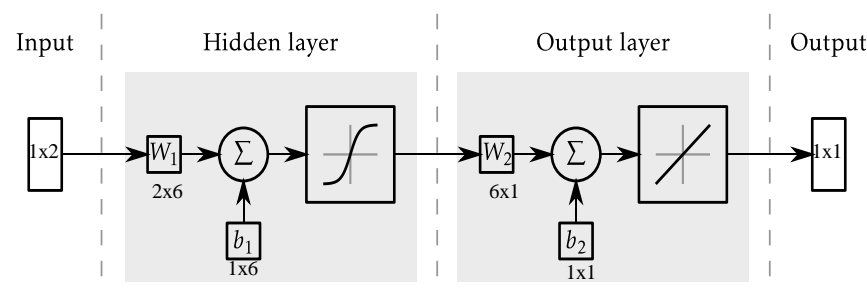

Fig. 12. The two-layer neural network structure used for the brake models, Size of the input/output and hidden and output layers are shown by matrix dimensions in the figure.

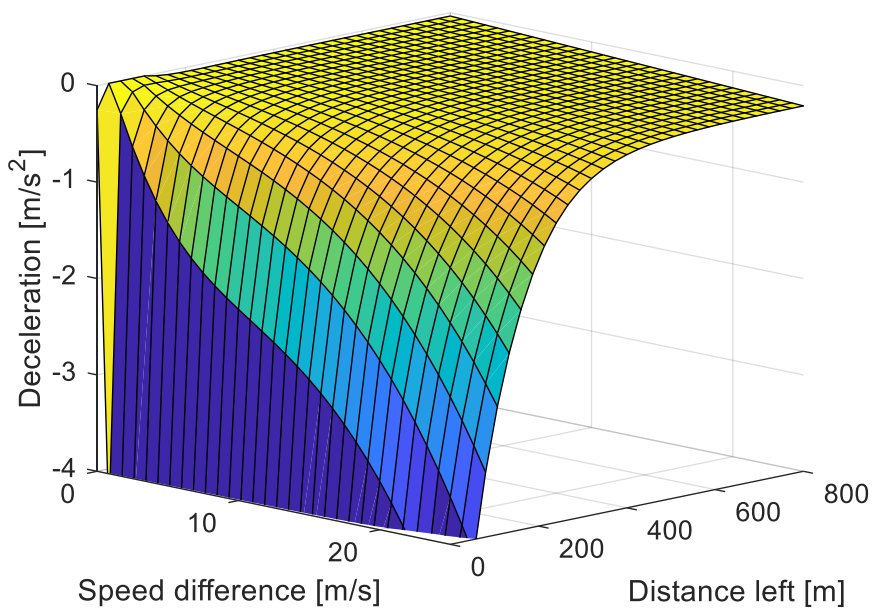

Fig. 13. Braking profile model for curves

\section{G. Acceleration Model}

A simple acceleration model was implemented, based on assumption that the maximum achievable acceleration, $a_{g}$ depends on the gear the vehicle is running on. The gear shifting algorithm initiates a gear change when the speed goes above values defined for gear changing, $v_{g c}$, one for each gear. Some sample acceleration sequences from one of the test persons was used to set the parameter values:

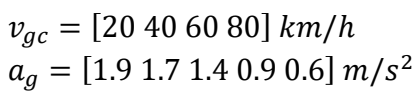

Additionally, when the current speed value is over $85 \%$ of the desired speed, the acceleration level will start to decrease gradually.

\section{EFFECT OF BEHAVIORAL MODELS ON RESULTING DRIVING CYCLES}

The two selected driving missions, Route 1 and Route 2, were simulated in SUMO and compared with the gathered data on the road. The achieved results show that using the developed behavioral models improve the match between simulation results and real driving data significantly. This is further discussed in the following sections.

\section{A. Assessment of the Improvements with Visual Inspection}

Fig. 14 and Fig. 15 show the simulation results of the two selected driving missions, Route 1 and Route 2, using the default behavior model in SUMO and the extended behavioral model. The gathered data on the road is also overlaid for comparison. It can be seen that the simulation outputs match the measurements significantly better with the extended behavioral model. Both braking and acceleration match the measurements on the road better with the extended behavioral model compared with the default model. The speed adaptation in a curve and speed variation during free driving resemble the natural driving on the road better when the extended behavioral model is used in the traffic simulation.

If needed, the match between the driving data and simulation results can be further enhanced by improving the models and using more driving data for training them. However, this is not critical for the purpose of this study, since the objective is not to develop a perfect behavioral model, but to illustrate how a traffic simulation tool can be used for generation of missionbased driving cycles, and to demonstrate the effects of an improved behavioral model on the resulting driving cycles. 


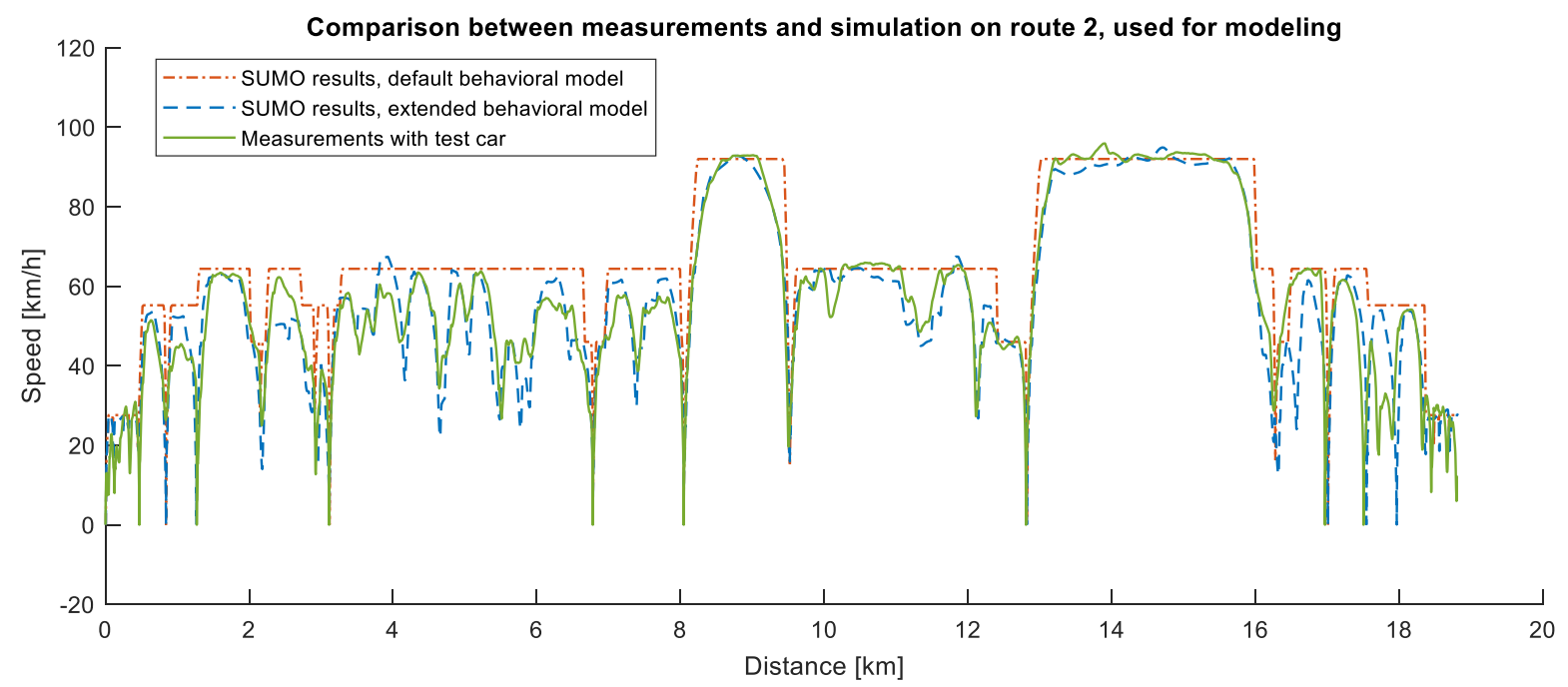

Fig. 14. The measured speed on Route 2 versus the traffic simulation output, before and after implemented changes in the behavioral model. The measured data on Route 2 was used for parameterizing the developed extensions of the behavioral model.

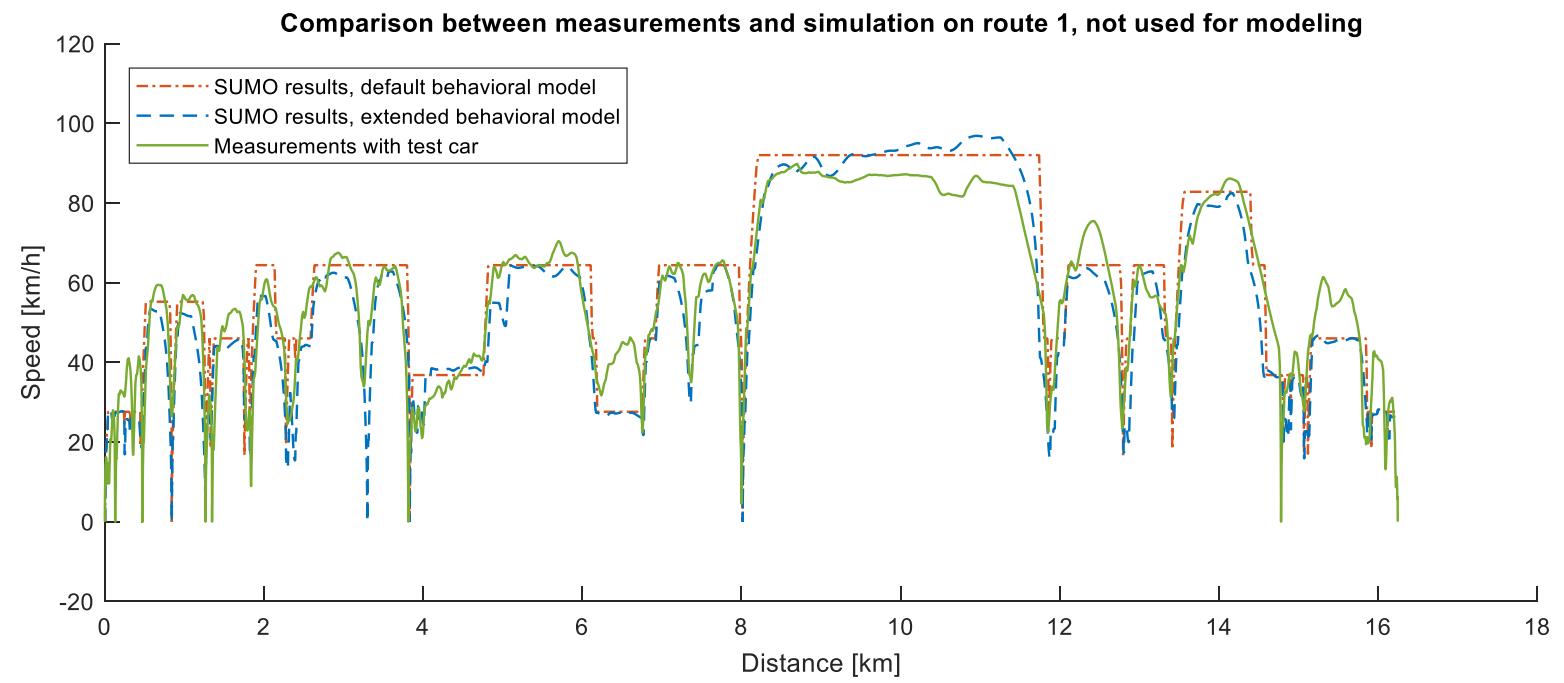

Fig. 15. The measured speed on Route 1 versus the traffic simulation output, before and after implemented changes in the behavioral model. The measured data on Route 1 was not used in parameterizing the developed extensions of behavioral models.

\section{B. Assessment of the Improvements with Objective Measures}

To further assess the achieved improvements in the resulting driving cycles by using the modified behavioral model, a few objective measures were used. In a research conducted by Ericsson [27], a list of driving pattern parameters correlated with the fuel consumption and emissions are provided. Three of these parameters, namely average acceleration (for acceleration phases where $a>0.1$ ), average deceleration acceleration (for deceleration phases where $a<-0.1$ ) and Relative Positive Acceleration (RPA) were selected for comparison of the resulting mission-based driving cycles from SUMO simulations. RPA is a parameter representing the occurrence of accelerations which demand large amount of power and is calculated as:

$$
\begin{aligned}
& R P A=\frac{1}{x} \int v(t) a^{+}(t) d t \\
& a^{+}(t)=\left\{\begin{array}{cc}
a(t) & a(t)>0.1 \\
0 & \text { otherwise }
\end{array}\right.
\end{aligned}
$$

where $x$ is the total distance, and $v$ and $a$ are speed and acceleration, respectively.

To analyze the effect of the improved behavioral models on the resulting mission-based driving cycles, the RPA, average acceleration and average deceleration for the driving cycle representing the driving mission on Route 2 were calculated and compared, see Table 1. Note that the values provided for the measurements on road is a mean value for all the drivers. The results from the traffic simulation with the extended behavioral model are closer to the measured values on road, compared to the results from simulation with the default behavioral model. The average deceleration in the simulation with default behavioral model is 4 times larger than the measured data, but this difference is reduced down to $10 \%$ using the extended behavioral model. Furthermore, the $40 \%$ difference in RPA of the originally simulated driving cycle compared to measured data is eliminated by the extended model. The improvement in the average acceleration measure is less than the other two; this can be due to the fact that the added acceleration model is too 
simple. Nevertheless, the achieved improvements indicate the value of development of more realistic behavioral models for generation of mission-based driving cycles by traffic simulation.

Table 1. RPA and average acceleration and deceleration measured on new route, measurements on the road versus traffic simulation.

\begin{tabular}{|l|c|c|c|}
\hline & RPA & Average acc. & Average dec. \\
\hline $\begin{array}{l}\text { Measurements on road } \\
\text { (mean value for all drivers) }\end{array}$ & 0.19 & 0.58 & -0.54 \\
\hline $\begin{array}{l}\text { Traffic simulation with the } \\
\text { default behavioral model }\end{array}$ & 0.11 & 1.65 & -2.77 \\
\hline $\begin{array}{l}\text { Traffic simulation with the } \\
\text { extended behavioral model }\end{array}$ & 0.19 & 1.17 & -0.59 \\
\hline
\end{tabular}

\section{CONCLUSIONS}

Feasibility of generation of mission-based driving cycles by merging traffic simulation and driver modelling was investigated and encouraging results were achieved. Comparison between real driving data and traffic simulation results showed that traffic simulation can be used to generate mission-based driving cycles with similar driving patterns to real driving data. It was also illustrated that the match between the resulting mission-based driving cycles and real driving data can be improved by extending the exiting behavioral model in the traffic simulation tool, staying within the same class of computational complexity of models. The added extensions to the behavioral model are: speed adaptation to the road curvature, natural speed variation in free driving, gradual braking behavior for preserving energy, and a speed/gear dependent acceleration. The extensions to the behavioral model were developed and parameterized based on driving data gathered on road, using e.g. machine learning algorithms. The obtained results show that even using limited real driving data results in significant improvements, observed by visual inspection and measured by objective measures.

\section{REFERENCES}

[1] P. Bielaczyc, J. Woodburn, and A. Szczotka, "A comparison of carbon dioxide exhaust emissions and fuel consumption for vehicles tested over the NEDC, FTP-75 and WLTC chassis dynamometer test cycles," SAE Technical Paper, 2015-01-1065, 2015.

[2] M. Tutuianu, et al., "Development of the World-wide harmonized Light duty Test Cycle (WLTC) and a possible pathway for its introduction in the European legislation," Transportation Research Part D, vol. 40, pp. 61-75, 2015.

[3] M. Andre, "The ARTEMIS European driving cycles for measuring car pollutant emissions," Science of the Total Environment, vol. 334, pp. 7384, 2004.

[4] J. Lin, and D. A. Niemeier, "An exploratory analysis comparing a stochastic driving cycle to California's regulatory cycle," Atmospheric Environment, vol. 36, pp. 5759-5770, 2002.

[5] T. Lee, and Z. S. Filipi, "Synthesis of real-world driving cycles using stochastic process and statistical methodology," International Journal of Vehicle Design, vol. 57, no.1, pp. 17-35, 2011.

[6] E. Shahedinejad, E. Bibeau, and S. Filizadeh, "Statistical development of a duty cycle for plug-in vehicles in a north American urban setting using fleet information," IEEE Transactions on Vehicular Technology, vol. 59, no. 8, pp. 3710-3719, 2010.

[7] P. Nyberg, E. Frisk, and L. Nielsen, "Using real-world driving databases to generate driving cycles with equivalence properties," IEEE Transactions on Vehicular Technology, vol. 65, no. 6, pp. 4095-4105,
2016

[8] P. Nyberg, E. Frisk, and L. Nielsen, "Driving cycle equivalence and transformation," IEEE Transactions on Vehicular Technology, vol. 66, no. 3, pp. 1963-1974, 2017.

[9] E. Silvas, K. Hereijgers, Huei Peng, T. Hofman, and M. Steinbuch, "Synthesis of realistic driving cycles with high accuracy and computational speed, including slope information", IEEE Transactions on Vehicular Technology, vol. 65, no. 6, pp. 4118-4128, 2016.

[10] Z. Liu, A. Ivanco, and Z. S. Filipi, "Naturalistic driving cycle synthesis by Markov chain of different orders", International Journal of Powertrains, vol. 6, no. 4, pp. 307-322, 2018.

[11] A. Esser, M. Zeller, S. Foulard, and S. Rinderknecht, "Stochastic synthesis of representative and multidimensional driving cycles," $S A E$ Technical Paper, 2018-01-0095, 2018.

[12] G. Amirjamshidi, and M. J. Roorda, "Development of simulated driving cycles for light, medium, and heavy duty trucks: Case of the Toronto Waterfront Area," Transportation Research Part D, vol. 34, pp. 255-266, 2015.

[13] G. Song, L. Yu, and Y. Zhang, "Applicability of traffic microsimulation models in vehicle emissions estimates, Case study of VISSIM," Transportation Research Record: Journal of the TRB, vol. 2270, pp. 132$141,2012$.

[14] A. Tapani, "Traffic simulation modelling of rural roads and driver assistance systems," PhD dissertation, No. 1211, Linköping University, 2008.

[15] J. Olstam, "Simulation of surrounding vehicles in driving simulations," PhD dissertation, No. 1248, Linköping University, 2009.

[16] T. Toledo, "Driving behaviour models and challenges," Transport Review: A Transnational Transdisciplinary Journal, vol. 27, no. 1, pp. 65-84, 2007.

[17] M. Behrisch, L. Bieker, J. Erdmann, and D. Krajzewicz, "SUMO Simulation of Urban Mobility," in Proc. of the third international conference on advances in system simulation, Barcelona, Spain, 2011.

[18] S. Krauß, "Microscopic modeling of traffic flow: Investigation of collision free vehicle dynamics," PhD dissertation, University of Cologne, 1998.

[19] J. C. McCall, D. P. Wipf, M. M. Trivedi, and B. D. Rao, "Lane change intent analysis using robust operators and sparse Bayesian learning," IEEE Transactions on Intelligent Transportation Systems, vol. 8, no. 3, pp. 431-440, 2007.

[20] F. Tango, L. Minin, F. Tesauri, and R. Montanari, "Field tests and machine learning approaches for refining algorithms and correlations of driver's model parameters," Applied Ergonomics, vol. 41, pp. 211-224, 2010.

[21] A. Wegener, M. Piorkowski, M. Raya, H. Hellbrück, S. Fischer and J. Hubaux, "TraCI: a framework for coupling road traffic and network simulators.," in Proc. of the 11th Communications and Networking Simulation Symposium, 2008

[22] T. Hida, "Brownian motion", in Brownian Motion, Springer, 1980, pp. 44-113.

[23] S. Haykin, Neural Networks: A Comprehensive Foundation. Prentice Hall PTR, 1994.

[24] K. Hornik, M. Stinchcombe, and H. White, H. "Multilayer feedforward networks are universal approximators," Neural networks, vol. 2. No. 5, pp. 359-366, 1998.

[25] K. Levenberg, "A method for the solution of certain non-linear problems in least squares," Quarterly of applied mathematics, vol. 2, no. 2, pp.164$168,1944$.

[26] D. W. Marquardt, "An algorithm for least-squares estimation of nonlinear parameters," Journal of the society for Industrial and Applied Mathematics, vol. 11, no. 2, pp. 431-441, 1963.

[27] E. Ericsson, "Independent driving pattern factors and their influence on fuel use and exhaust emission factors," Transportation Research Part D, vol. 6. pp. 325-345, 2001. 


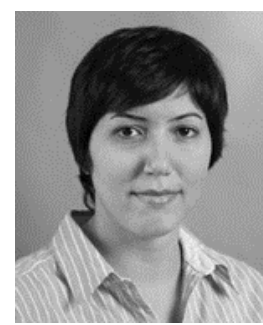

Sogol Kharrazi was born in Iran in 1980. She received the B.Sc. degree in mechanical engineering from Sharif University of Technology, Tehran, Iran, and M.Sc. degree in automotive engineering from Chalmers University of Technology, Gothenburg, Sweden. She received the $\mathrm{PhD}$ degree in machine and vehicle systems from Chalmers University of Technology, Gothenburg, Sweden, in 2012.

She is currently an adjunct associate professor at Department of Electrical Engineering, Linköping University, Linköping, Sweden. She also works as a researcher at the Swedish National Road and Transport Research Institute, Linköping, Sweden. Her research interests include vehicle dynamics and control and driving behavior modeling.

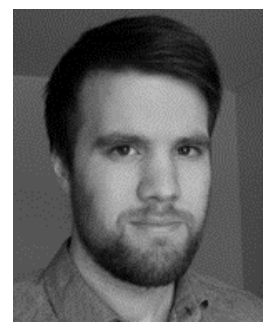

Marcus Almén was born in Sweden in 1993. He received the M.Sc. degree in electrical engineering from Linköping University, Linköping, Sweden in 2017.

$\mathrm{He}$ is currently working as a system engineer at Saab Defense \& Space, Linköping, Sweden. His research interests include modeling, simulation and control.

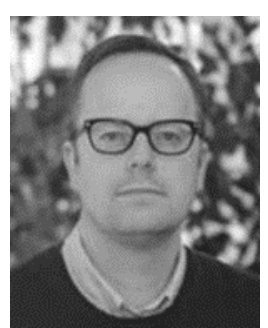

Erik Frisk was born in Sweden in 1971. $\mathrm{He}$ received the Ph.D. degree from Linköping University, Linköping, Sweden, in 2001.

$\mathrm{He}$ is currently a Professor with the Department of Electrical Engineering, Linköping University. His research interests include issues in model-based fault diagnosis and fault isolation, prognostics, and observer theory.

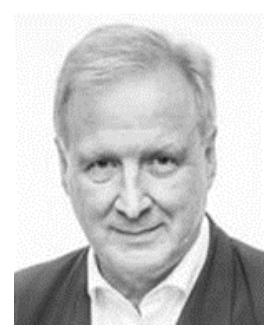

Lars Nielsen was born in Sweden in 1955. He received the M.Sc. degree in engineering physics and the Ph.D. degree in automatic control from Lund Institute of Technology, Lund, Sweden, in 1979 and 1985, respectively.

Since 1992, he has been a Professor with the Division of Vehicular Systems, Department of Electrical Engineering, Linköping University, Linköping, Sweden, where he holds the Sten Gustafsson Chair. His research interests include automotive modeling, control, diagnosis, and autonomous functions. 